\title{
IDENTITIES INVOLVING THE COEFFICIENTS OF A CLASS OF DIRICHLET SERIES. III
}

\author{
BY \\ BRUCE C. BERNDT
}

1. Introduction and summary of results. This paper is a continuation of the study of identities involving arithmetical functions generated by a class of Dirichlet series satisfying functional equations with $\Gamma$-factors. The study is primarily a sequel to [1], [2], [5], [7].

Before proceeding further, we establish some notation. We let $s=\sigma+i$ with $\sigma$ and $t$ both real. We write $\int_{(c)}$ for $\int_{c-i \infty}^{c+i \infty}$ and $\sum$ for $\sum_{n=1}^{\infty} . \mathscr{R}\left(f(s), s_{0}\right)$ denotes the residue of $f(s)$ at $s=s_{0} . I(C)$ denotes the interior of a closed curve, or curves. $c, c_{1}, c_{2}$ and $A>0$ denote constants, not necessarily the same with each occurrence.

The series in question are given by the following

Definition. Let $\left\{\lambda_{n}\right\}$ and $\left\{\mu_{n}\right\}$ be two sequences of positive numbers strictly increasing to $\infty$, and $\{a(n)\}$ and $\{b(n)\}$ two sequences of complex numbers not identically zero. Consider the functions $\varphi$ and $\psi$ representable as Dirichlet series

$$
\varphi(s)=\sum a(n) \lambda_{n}^{-s}, \quad \psi(s)=\sum b(n) \mu_{n}^{-s}
$$

with finite abscissas of absolute convergence $\sigma_{a}$ and $\sigma_{a}^{*}$, respectively. For any positive integer $N$, let

$$
\Delta(s)=\prod_{k=1}^{N} \Gamma\left(\alpha_{k} s+\beta_{k}\right),
$$

where $\alpha_{k}>0$ and $\beta_{k}$ is complex, $k=1, \ldots, N$. If $r$ is real, we say that $\varphi$ and $\psi$ satisfy the functional equation

$$
\Delta(s) \varphi(s)=\Delta(r-s) \psi(r-s)
$$

if there exists in the s-plane a domain $D$ which is the exterior of a compact set $S$, such that in $D$ a holomorphic function $\chi$ exists with the properties:

(i) $\chi(s)=\Delta(s) \varphi(s), \sigma>\sigma_{a}, \chi(s)=\Delta(r-s) \psi(r-s), \sigma<r-\sigma_{a}^{*}$;

(ii) $\lim _{|t| \rightarrow \infty} \chi(\sigma+i t)=0$, uniformly in every interval $-\infty<\sigma_{1} \leqq \sigma \leqq \sigma_{2}<\infty$.

It can be shown that (1.1) and the relation

$$
\sum a(n) \int_{(c)} \Delta(s)\left(\lambda_{n} y\right)^{-s} d s=y^{-r} \sum b(n) \int_{(c)} \Delta(s)\left(\mu_{n} / y\right)^{-s} d s+2 \pi i P(y)
$$

Received by the editors March 17, 1969. 
are equivalent, where $c, y>0$ and

$$
P(y)=\frac{1}{2 \pi i} \int_{C} \chi(s) y^{-s} d s,
$$

where $C$ is a curve, or curves, containing $S$. If $\Delta(s)=\Gamma(s)$, the proof is given in [4]. If $\Delta(s)=\Gamma^{N}(s)$, the proof is given in [1], and the proof in general follows along the same lines. In particular, if $\Delta(s)=\Gamma(s)$,

$$
\Phi(y)=\sum a(n) \exp \left(-\lambda_{n} y\right), \quad \Psi(y)=\sum b(n) \exp \left(-\mu_{n} y\right),
$$

then Hecke's functional equation

$$
\Gamma(s) \varphi(s)=\Gamma(r-s) \psi(r-s)
$$

and the "modular relation"

$$
\Phi(y)=y^{-r} \Psi(1 / y)+P(y)
$$

are equivalent, upon the use of (2.5) below. There are a few other cases when (1.2) can be expressed in terms of elementary functions. Thus, using (2.14) below, we find that $\varphi$ and $\psi$ satisfy

$$
\Gamma(s+v / 2) \Gamma(s-v / 2) \varphi(s)=\Gamma(r-s+v / 2) \Gamma(r-s-v / 2) \psi(r-s)
$$

if and only if

$$
2 \sum a(n) K_{v}\left(2\left\{\lambda_{n} y\right\}^{1 / 2}\right)=2 y^{-r} \sum b(n) K_{v}\left(2\left\{\mu_{n} / y\right\}^{1 / 2}\right)+P(y),
$$

where $K_{v}$ is the modified Bessel function usually so denoted and $v$ is an arbitrary complex number.

In $\S \S 3-6$ we consider four special cases of (1.1). Our aim is to develop identities for the Riesz sum

and the logarithmic sum

$$
A_{q}(x)=\frac{1}{\Gamma(q+1)} \sum_{\lambda_{n} \leqq x} a(n)\left(x-\lambda_{n}\right)^{q}
$$

$$
S(x ; q)=\frac{1}{\Gamma(q+1)} \sum_{\lambda_{n} \leqq x} a(n) \log ^{q}\left(x / \lambda_{n}\right),
$$

where $q \geqq 0$ is subject to certain other restrictions. Identities in other special cases have been given in [1], [2], [5], [7].

$\$ \$ 7-10$ are concerned with other types of identities for coefficients of series satisfying (1.3). In $\$ 7$ an identity analogous to (1.4) involving the exponential integral is established. In [3] we define a generalized Dirichlet series that is analogous to the generalized zeta-function of Riemann. We derive a formula for such functions which enables us to obtain an analytic continuation for them and also to determine a few simple properties. In $\S 8$ we give an easier proof of this formula, but in addition show that it implies (1.3). In $\S 9$ we establish an identity involving the Bessel 
function $K_{v}(x)$. A particular case of this identity is due to Ramanujan. $\$ 10$ gives identities involving Laguerre polynomials.

Most of our identities are completely new; some are generalizations of identities proven for special arithmetical functions. We conclude with some examples in $\S 11$.

2. Preliminary results. We shall need several results from the theories of the $\Gamma$-function and the Bessel functions $J_{v}, Y_{v}$ and $K_{v}$. We also require the values of a number of definite integrals.

We have

$$
|\Gamma(s)| \sim(2 \pi)^{1 / 2}|t|^{\sigma-1 / 2} \exp (-\pi|t| / 2),
$$

uniformly for $-\infty<\sigma_{1} \leqq \sigma \leqq \sigma_{2}<\infty$ [8, p. 224]. From [8, p. 211]

$$
\Gamma(s) \Gamma(1-s)=\pi / \sin (\pi s)
$$

and $[8$, p. 212]

$$
\Gamma(s) \Gamma(s+1 / 2)=2^{1-2 s} \pi^{1 / 2} \Gamma(2 s)
$$

we easily deduce that

$$
\Gamma(s / 2) / \Gamma(\{1-s\} / 2)=2^{1-s} \pi^{-1 / 2} \cos (\pi s / 2) \Gamma(s) .
$$

For $x, c>0[10$, p. 312],

$$
\frac{1}{2 \pi i} \int_{(c)} \Gamma(s) x^{-s} d s=e^{-x}
$$

For $x>0$ and $0<c<1[10$, p. 348],

$$
\frac{1}{2 \pi i} \int_{(c)} \Gamma(s) \cos (\pi s / 2) x^{-s} d s=\cos x
$$

The exponential integral Ei $(-x), x>0$, may be defined by [11, p. 925]

$$
\operatorname{Ei}(-x)=-\int_{x}^{\infty} \frac{e^{-t}}{t} d t
$$

If $\sigma>0$,

$$
\begin{aligned}
\int_{0}^{\infty} \operatorname{Ei}(-x) x^{s-1} d x & =-\int_{0}^{\infty} x^{s-1} d x \int_{x}^{\infty} \frac{e^{-t}}{t} d t \\
& =-\int_{0}^{\infty} \frac{e^{-t}}{t} d t \int_{0}^{t} x^{s-1} d x=-\frac{\Gamma(s)}{s},
\end{aligned}
$$

and by Mellin's inversion formula for $c>0$,

$$
\operatorname{Ei}(-x)=-\frac{1}{2 \pi i} \int_{(c)} \frac{\Gamma(s)}{s} x^{-s} d s .
$$

For $\operatorname{Re} \beta, \operatorname{Re} \gamma>0$ and real $p \neq 0$ [11, p. 342],

$$
\int_{0}^{\infty} x^{\nu-1} \exp \left(-\beta x^{p}-\gamma x^{-p}\right) d x=\frac{2}{p}\left(\frac{\gamma}{\beta}\right)^{v / 2 p} K_{v / p}\left(2\{\beta \gamma\}^{1 / 2}\right) .
$$


For $x, \operatorname{Re} a>0[10$, p. 361],

$$
\frac{1}{2 \pi i} \int_{(c)} K_{s}(a) x^{-s} d s=\frac{1}{2} e^{-a(x+1 / x) / 2} .
$$

For $0<c<\operatorname{Re} v / 2+3 / 4[10$, p. 326],

$$
J_{v}(x)=\frac{1}{2 \pi i} \int_{(c)} \frac{\Gamma(s)}{\Gamma(\nu+1-s)}\left(\frac{x}{2}\right)^{v-2 s} d s .
$$

For $0 \leqq \operatorname{Re} \nu<c<\operatorname{Re} v / 2+3 / 4<3 / 2$ [10, p. 329],

$$
-\pi Y_{\nu}(x)=\frac{1}{2 \pi i} \int_{(c)} \Gamma(s) \Gamma(s-v) \cos \{\pi(s-v)\}\left(\frac{x}{2}\right)^{v-2 s} d s .
$$

For $0 \leqq \operatorname{Re} \nu<c[10$, p. 331$]$,

$$
2 K_{v}(x)=\frac{1}{2 \pi i} \int_{(c)} \Gamma(s) \Gamma(s-\nu)\left(\frac{x}{2}\right)^{\nu-2 s} d s .
$$

We have the following asymptotic formulas [18, pp. 198-202] as $z$ tends to $\infty$,

$$
\begin{aligned}
& J_{v}(z)=\left(c_{1} e^{i z}+c_{2} e^{-i z}\right) z^{-1 / 2}+O\left(z^{-3 / 2}\right), \\
& Y_{v}(z)=\left(c_{1} e^{i z}+c_{2} e^{-i z}\right) z^{-1 / 2}+O\left(z^{-3 / 2}\right),
\end{aligned}
$$

and

$$
K_{v}(z)=e^{-z}\left\{c z^{-1 / 2}+O\left(z^{-3 / 2}\right)\right\} .
$$

From [18, pp. 45, 66 and 79],

$$
\begin{gathered}
d\left\{z^{v} J_{v}(z)\right\} / d z=z^{v} J_{v-1}(z), \\
d\left\{z^{v} Y_{v}(z)\right\} / d z=z^{v} Y_{v-1}(z),
\end{gathered}
$$

and

$$
d\left\{z^{v} K_{v}(z)\right\} / d z=-z^{v} K_{v-1}(z)
$$

Also [18, p. 80],

$$
K_{1 / 2}(z)=(\pi / 2 z)^{1 / 2} e^{-z}
$$

The Laguerre polynomial $L_{n}^{(\alpha)}(x)$ may be defined by [11, p. 1037]

$$
L_{n}^{(\alpha)}(x)=\frac{1}{n !} e^{x} x^{-\alpha} \frac{d^{n}}{d x^{n}}\left(e^{-x} x^{n+\alpha}\right) .
$$

We shall need the following lemmas in the sequel.

Lemma 2.1. Let

$$
r(x, y ; n)=\left(x^{-2}-y^{-2}\right)^{n} / n ! .
$$


Suppose $\xi_{1}, \ldots, \xi_{n}, y>0$ and $a<1 / 2$. Then,

$$
\begin{aligned}
\int_{y}^{\infty} \xi_{n}^{-3} d \xi_{n} \int_{\xi_{n}}^{\infty} \xi_{n-1}^{-3} d \xi_{n-1} \cdots \int_{\xi_{2}}^{\infty} \xi_{1}^{-3} d \xi_{1} \int_{\xi_{1}}^{\infty} x^{a} J_{v}(x) d x \\
=\frac{(-1)^{n}}{2^{n}} \int_{y}^{\infty} x^{a} J_{v}(x) r(x, y ; n) d x .
\end{aligned}
$$

Proof. We proceed by induction. For $n=0$ the conclusion is trivial. Assuming that the result is true for $n-1$, we are led to examine

$$
\begin{aligned}
\frac{(-1)^{n-1}}{2^{n-1}} \int_{y}^{\infty} \xi_{n}^{-3} d \xi_{n} & \int_{\xi_{n}}^{\infty} x^{a} J_{v}(x) r\left(x, \xi_{n} ; n-1\right) d x \\
& =\frac{(-1)^{n-1}}{2^{n-1}} \int_{y}^{\infty} x^{a} J_{v}(x) d x \int_{y}^{x} \xi_{n}^{-3} r\left(x, \xi_{n} ; n-1\right) d \xi_{n},
\end{aligned}
$$

where the inversion in order of integration is justified by a theorem in [13, p. 349]. The result now follows by evaluating the inner integral.

Define (as in [2]) for $x, y>0$

$$
s(x, y ; n)=(-1)^{n} \log ^{n}(x / y) / n ! .
$$

Note that for $n>0$,

$$
\partial s(x, y ; n) / \partial y=s(x, y ; n-1) / y
$$

and

$$
s(y, y ; n)=0 .
$$

Lemma 2.2. Suppose $\xi_{1}, \ldots, \xi_{n}, n>0$ and $a<0$. Then,

$$
\int_{y}^{\infty} \xi_{n}^{-1} d \xi_{n} \cdots \int_{\xi_{2}}^{\infty} \xi_{1}^{-1} d \xi_{1} \int_{\xi_{1}}^{\infty} x^{a} e^{ \pm i x} d x=(-1)^{n} \int_{y}^{\infty} x^{a} e^{ \pm i x} s(x, y ; n) d x .
$$

The proof of Lemma 2.2 follows along the same lines as that of Lemma 2.1 and can be found in [2, Lemma 2.7] where the result was stated for a different integrand.

Lemma 2.3. Let $\sigma<0$ and $t$ be fixed. Then, for $a \geqq 0$

$$
I=\int_{\xi}^{\infty} x^{s} e^{ \pm i x} \log ^{a} x d x=O\left(\xi^{\sigma} \log ^{a} \xi\right)
$$

as $\xi$ tends to $\infty$.

Proof. It is clearly sufficient to examine the integral involving $e^{i x}$. Without loss of generality we may assume that $\xi$ is large enough so that $\left(x^{\sigma} \log ^{a} x\right) /(1+t / x)$ is monotonic on $(\xi, \infty)$. Write

$$
\begin{aligned}
I & =\int_{\xi}^{\infty} x^{\sigma} \exp (i(x+t \log x)) \log ^{a} x d x \\
& =\int_{\xi}^{\infty} x^{\sigma} \log ^{a} x \cos u d x+i \int_{\xi}^{\infty} x^{\sigma} \log ^{a} x \sin u d x \\
& =I_{1}+I_{2}
\end{aligned}
$$


where $u=x+t \log x$. Since $d u / d x=1+t / x>0$ for $\xi$ large enough, $x$ and $u$ increase together. Thus, by the second mean value theorem for integrals [17, p. 379],

$$
\begin{aligned}
I_{1} & =\int_{x=\xi}^{x=\infty} \frac{x^{\sigma} \log ^{a} x \cos u}{1+t / x} d u \\
& =\frac{\xi^{\sigma} \log ^{a} \xi}{1+t / \xi} \int_{x=\xi}^{x=\xi^{\prime}} \cos u d u=O\left(\xi^{\sigma} \log ^{a} \xi\right),
\end{aligned}
$$

where $\xi^{\prime}<\infty . I_{2}$ is treated similarly, and we are done.

3. The functional equation $\Gamma(\{s+1\} / 2) \varphi(s)=\Gamma(\{2-s\} / 2) \psi(1-s)$. To derive our identity for $A_{q}(x)$ we need the following

LEMMA 3.1. Let $\nu$ be a positive integer and $0<c<\nu-1 / 2$, where $c$ is not an odd integer. Then, for $x>0$,

where

$$
\frac{1}{2 \pi i} \int_{(c)} \frac{x^{-s}}{\Gamma(v+1-s) \cos (\pi s / 2)} d s=\frac{2}{\pi} T_{v}(x)
$$

$$
\begin{array}{ll}
T_{\nu}(x)=\frac{(-1)^{v / 2}}{x^{v}}\left(\sin x-\sum_{k=0}^{[(\nu-c-1) / 2]} \frac{(-1)^{k} x^{2 k+1}}{(2 k+1) !}\right), \quad \text { v even, } \\
T_{\nu}(x)=\frac{(-1)^{(v+1) / 2}}{x^{\nu}}\left(\cos x-\sum_{k=0}^{[(v-c) / 2]} \frac{(-1)^{k} x^{2 k}}{(2 k) !}\right), \quad v \text { odd },
\end{array}
$$

where if $v-c-1<0$, the sum is empty.

Proof. We have from (2.1),

$$
1 / \Gamma(\nu+1-s) \cos (\pi s / 2)=O\left(|t|^{\sigma-\nu-1 / 2}\right) .
$$

Thus, the hypothesis $c<v-1 / 2$ secures the convergence of the integral.

Consider

$$
I=\frac{1}{2 \pi i} \int_{C} \frac{x^{-s}}{\Gamma(\nu+1-s) \cos (\pi s / 2)} d s
$$

where $C$ denotes the rectangle with vertices $c-i R, c+i R,-2 m+i R$ and $-2 m-i R$, where $m$ is a positive integer and $R>0$. On $I(C)$ the integrand has simple poles at $s=-2 m+1,-2 m+3, \ldots,-1,1, \ldots, 2[(c-1) / 2]+1$. The residue at $s=2 n+1$ is $-2(-1)^{n} x^{-2 n-1} / \pi \Gamma(\nu-2 n)$. Hence,

$$
I=-\frac{2}{\pi} \sum_{-m \leqq n<(c-1) / 2} \frac{(-1)^{n} x^{-2 n-1}}{\Gamma(\nu-2 n)} .
$$

Now, (3.1) holds uniformly on $-2 m \leqq \sigma \leqq c$. Thus, the integrals along the horizontal sides of $C$ tend to 0 as $R$ tends to $\infty$. Hence, we also have

$$
I=\frac{1}{2 \pi i}\left(\int_{(c)}-\int_{(-2 m)}\right) \frac{x^{-s}}{\Gamma(\nu+1-s) \cos (\pi s / 2)} d s .
$$


Since

$$
\Gamma(\nu+1+2 m-i t)=(\nu+2 m-i t) \cdots(2-i t) \Gamma(2-i t),
$$

we conclude from (2.1) that

$$
|\Gamma(\nu+1+2 m-i t)| \geqq A(\nu+2 m) !(|t|+1)^{3 / 2} \exp (-\pi|t| / 2) .
$$

Hence,

$$
\left|\int_{(-2 m)} \frac{x^{-s}}{\Gamma(\nu+1-s) \cos (\pi s / 2)} d s\right| \leqq \frac{A x^{2 m}}{(\nu+2 m) !} \int_{-\infty}^{\infty} \frac{d t}{(|t|+1)^{3 / 2}}=o(1),
$$

as $m$ tends to $\infty$. Thus, (3.2) and (3.3) yield

$$
\begin{aligned}
\frac{1}{2 \pi i} \int_{(c)} \frac{x^{-s}}{\Gamma(\nu+1-s) \cos (\pi s / 2)} d s & =-\frac{2}{\pi} \sum_{n>(1-c) / 2} \frac{(-1)^{n} x^{2 n-1}}{\Gamma(\nu+2 n)} \\
& =\frac{2}{\pi} T_{\nu}(x) .
\end{aligned}
$$

THEOREM 3.2. Let $\varphi$ satisfy the functional equation

$$
\Gamma(\{s+1\} / 2) \varphi(s)=\Gamma(\{2-s\} / 2) \psi(1-s) .
$$

Suppose that $x>0$ and $q$ is a nonnegative integer.

(i) Let $\gamma>\sup \left(0, \sigma_{a}, \sigma_{a}^{*}\right)$ where $\gamma$ is not an odd integer. Then if $T_{\nu}$ is given as in Lemma 3.1 and $q>\gamma-1 / 2$,

$$
A_{q}(x)=Q_{q}(x)+2 \pi^{-1 / 2} x^{q+1} \sum b(n) T_{q+1}\left(2 \mu_{n} x\right),
$$

where

$$
Q_{q}(x)=\frac{1}{2 \pi i} \int_{C} \frac{\Gamma(s) \varphi(s) x^{s+q}}{\Gamma(s+q+1)} d s,
$$

where $C$ is a curve, or curves, encircling the singularities of the integrand in the strip $1-\gamma<\sigma<\gamma$.

(ii) Let $1<\gamma<2$ where $\gamma>\sup \left(0, \sigma_{a}, \sigma_{a}^{*}\right)$. Let $s(x, y ; n)$ be given by (2.23). Then, for $q \geqq 1$,

$$
S(x ; q)=R(x ; q)+\pi^{-1 / 2} \sum \frac{b(n)}{\mu_{n}} \int_{u_{n} x}^{\infty} \frac{\cos (2 u)}{u} s\left(u, \mu_{n} x ; q-1\right) d u
$$

where

$$
R(x ; q)=\frac{1}{2 \pi i} \int_{C} \frac{\varphi(s) x^{s}}{s^{q+1}} d s,
$$

where $C$ is a curve, or curves, encircling all of the integrand's singularities in the strip $1-\gamma<\sigma<\gamma$.

Proof of (i). Proceeding as in [1, proof of Theorem 2], we arrive at

$$
A_{q}(x)=Q_{q}(x)+x^{q+1} \sum b(n) \frac{1}{2 \pi i} \int_{(\gamma)} \frac{\Gamma(1-s) \Gamma(\{s+1\} / 2)\left(\mu_{n} x\right)^{-s}}{\Gamma(q+2-s) \Gamma(\{2-s\} / 2)} d s,
$$


provided that $q>\gamma-1 / 2$. Now, by (2.3) and (2.2),

$$
\begin{aligned}
\frac{\Gamma(1-s) \Gamma(\{s+1\} / 2)}{\Gamma(\{2-s\} / 2)} & =\frac{\Gamma(1-s) 2^{1-s} \pi^{1 / 2} \Gamma(s)}{\Gamma(\{2-s\} / 2) \Gamma(s / 2)} \\
& =\frac{2^{1-s} \pi^{1 / 2} \sin (\pi s / 2)}{\sin (\pi s)}=\frac{2^{-s} \pi^{1 / 2}}{\cos (\pi s / 2)}
\end{aligned}
$$

Upon using Lemma 3.1 in (3.6), we have immediately (3.4), and the proof is complete.

Proof of (ii). Proceeding as in [2, proof of Theorem 1], we find that

$$
S(x ; q)=R(x ; q)+\sum \frac{b(n)}{\mu_{n}} \frac{1}{2 \pi i} \int_{(\gamma)} \frac{\Gamma(\{s+1\} / 2)\left(\mu_{n} x\right)^{1-s}}{\Gamma(\{2-s\} / 2)(1-s)^{q+1}} d s,
$$

where $q>\gamma-1 / 2$. Now, by (2.3) and (2.2),

$$
\begin{aligned}
\frac{\Gamma(\{s+1\} / 2)}{\Gamma(\{2-s\} / 2)(1-s)} & =\frac{2^{1-s} \pi^{-1 / 2} \Gamma(s) \sin (\pi s / 2)}{1-s} \\
& =-2^{1-s_{\pi}-1 / 2} \Gamma(s-1) \cos \{\pi(s-1) / 2\}
\end{aligned}
$$

Hence, by (2.6), since $1<\gamma<2$,

$$
\begin{aligned}
\frac{1}{2 \pi i} \int_{(\gamma)} \frac{\Gamma(\{s+1\} / 2) u^{-s}}{\Gamma(\{2-s\} / 2)(1-s)} d s & \\
= & -\frac{\pi^{-1 / 2}}{u} \frac{1}{2 \pi i} \int_{(\gamma)} \Gamma(s-1) \cos \{\pi(s-1) / 2\}(2 u)^{1-s} d s \\
& =-\frac{\pi^{-1 / 2} \cos (2 u)}{u} .
\end{aligned}
$$

Integrate both sides of (3.8) with respect to $u$ over $\left(\xi_{1}, \infty\right), \xi_{1}>0$. Inverting the order of integration by a theorem in [13, p. 349], we find that

$$
-\frac{1}{2 \pi i} \int_{(\gamma)} \frac{\Gamma(\{s+1\} / 2) \xi_{1}^{-s+1}}{\Gamma(\{2-s\} / 2)(1-s)^{2}} d s=-\pi^{-1 / 2} \int_{\xi_{1}}^{\infty} \frac{\cos (2 u)}{u} d u
$$

Multiply both sides of (3.9) by $1 / \xi_{1}$ and integrate with respect to $\xi_{1}$ over $\left(\xi_{2}, \infty\right)$, $\xi_{2}>0$. After $q$ such integrations we obtain

$$
\begin{aligned}
\frac{(-1)^{q}}{2 \pi i} \int_{(\gamma)} \frac{\Gamma(\{s+1) / 2) y^{-s+1}}{\Gamma(\{2-s\} / 2)(1-s)^{q+1}} d s & =-\pi^{-1 / 2} \int_{y}^{\infty} \frac{d \xi_{q-1}}{\xi_{q-1}} \cdots \int_{\xi_{1}}^{\infty} \frac{\cos 2 u}{u} d u \\
& =(-1)^{q} \pi^{-1 / 2} \int_{y}^{\infty} \frac{\cos (2 u)}{u} s(u, y ; q-1) d u
\end{aligned}
$$

by Lemma 2.2. Letting $y=\mu_{n} x$ and substituting the above into (3.7), we obtain (3.5) under the condition that $q>\gamma-1 / 2$. The identity may be upheld for $q \geqq 1$, however, as follows. By Lemma 2.3,

$$
\int_{\mu_{n} x}^{\infty} \frac{\cos (2 u)}{u} s\left(u, \mu_{n} x ; q-1\right) d u=O\left(\left\{\mu_{n} x\right\}^{-1} \log ^{q-1} \mu_{n} x\right) .
$$


Hence,

$$
\begin{aligned}
S(x ; q) & =R(x ; q)+O\left(\frac{1}{x} \sum \frac{|b(n)|}{\mu_{n}^{2}} \log ^{q-1} \mu_{n} x\right) \\
& =R(x ; q)+O\left(\left\{\log ^{q-1} x\right\} / x\right),
\end{aligned}
$$

as $\sigma_{a}^{*}<2$. Differentiating both sides of (3.5) with respect to $x$, we obtain, with the help of (2.24) and (2.25), (3.5) again, but with $q$ replaced by $q^{\prime}=q-1$. This differentiation is justified for $q^{\prime} \geqq 1$, since the above argument shows that the differentiated infinite series converges uniformly on any compact interval in $x>0$, provided that $q^{\prime} \geqq 1$.

4. The functional equation $\Gamma(s+m) \varphi(s)=\Gamma(r-s+m) \psi(r-s)$.

THEOREM 4.1. Let $\varphi$ satisfy the functional equation

$$
\Gamma(s+m) \varphi(s)=\Gamma(r-s+m) \psi(r-s),
$$

where $m$ is a positive integer. Suppose $x>0$ and let $\xi=2\left(\mu_{n} x\right)^{1 / 2}$.

(i) If $r(x, y ; n)$ is as in Lemma 2.1 and $q$ is a positive number such that

$$
q>\sup \left(2 \sigma_{a}^{*}-r-1 / 2, r+2 m-5 / 2\right),
$$

then

$$
\begin{aligned}
A_{q}(x)= & Q_{q}(x)-2^{q-r} \sum \frac{b(n)}{\mu_{n}^{r}} \frac{d^{m-1}}{d x^{m-1}} \\
& \cdot\left(x^{q+m-1} \int_{\xi}^{\infty} u^{r+2 m-q-2} J_{r+q+2 m-1}(u) r(u, \xi ; m-1) d u\right),
\end{aligned}
$$

where

$$
Q_{q}(x)=\frac{1}{2 \pi i} \int_{C} \frac{\Gamma(s) \varphi(s) x^{s+q}}{\Gamma(s+q+1)} d s
$$

where $C$ is a curve, or curves, encircling the singularities of the integrand.

(ii) Let $q$ be a positive integer such that $q>\sup \left(2 \sigma_{a}^{*}-r-1 / 2, r-1 / 2\right)$. Then,

$$
\begin{aligned}
S(x ; q)= & R(x ; q)-2^{2 q-r} x^{1-m} \sum \frac{b(n)}{\mu_{n}^{r}} \frac{d^{q}}{d x^{q}} \\
& \cdot\left(x^{q+m-1} \int_{\xi}^{\infty} u^{r-q} J_{r+q+2 m-1}(u) s(u, \xi ; q) d u\right),
\end{aligned}
$$

where

$$
R(x ; q)=\frac{1}{2 \pi i} \int_{C} \frac{\varphi(s) x^{s}}{s^{q+1}} d s,
$$

where $C$ is a curve, or curves, encircling the integrand's singularities.

We do not attempt to be exhaustive in the number of identities we can write down. Using a technique in [2, proof of Theorem 3], we may derive for smaller values of $q$ and with additional restrictions on $\varphi$ identities similar to (4.1) and (4.2). Also, using the method of equiconvergent trigonometric series [1], [5], we may 
extend the range of $q$ to $q>2 \sigma_{a}^{*}-r-3 / 2$, where $q$ may possibly be $\leqq 0$ as well. The proof of (ii) will be omitted as the proof is almost identical to that in $[2$, Theorem 1, (ii)].

Proof of (i). In the following we shall assume that $q$ is a complex variable. Let $\gamma$ be chosen large enough so that $\gamma>\sup \left(0, \sigma_{a}, \sigma_{a}^{*}, r+m-1\right)$ and so that $C$ lies in the strip $r-\gamma<\sigma<\gamma$. Proceeding as in [1, proof of Theorem 2], we find that for $\operatorname{Re} q>2 \gamma-r$,

$$
A_{q}(x)=Q_{q}(x)+x^{q} \sum \frac{b(n)}{\mu_{n}^{r}} \frac{1}{2 \pi i} \int_{(\gamma)} \frac{\Gamma(r-s) \Gamma(s+m)\left(\mu_{n} x\right)^{r-s}}{\Gamma(r-s+m) \Gamma(r+q+1-s)} d s .
$$

From (2.12) for $\operatorname{Re} q>2 \gamma-r-1 / 2$,

$$
\frac{1}{2 \pi i} \int_{(\gamma)} \frac{\Gamma(s+m)}{\Gamma(r+q+m-s)}\left(\frac{u}{2}\right)^{-2 s} d s=\left(\frac{u}{2}\right)^{-r-q+1} J_{r+q+2 m-1}(u)
$$

Multiply both sides of (4.4) by $u^{2 r+2 m-3}$ and integrate with respect to $u$ over $\left(\xi_{1}, \infty\right), \xi_{1}>0$. For the resulting integral on the right-hand side to converge, we require by (2.15) that $\operatorname{Re} q>r+2 m-5 / 2$. Inverting the order of integration [13, p. 349], we find that since $\gamma>r+m-1$,

$$
\begin{aligned}
-\frac{1}{2 \pi i} \int_{(\gamma)} \frac{2^{2 s} \Gamma(s+m) \xi_{1}^{2(r+m-s-1)}}{\Gamma(r+q+m-s)(r+m-s-1)} d s & \\
= & 2^{r+q} \int_{\xi_{1}}^{\infty} u^{r+2 m-q-2} J_{r+q+2 m-1}(u) d u .
\end{aligned}
$$

Now multiply both sides of (4.5) by $1 / \xi_{1}^{3}$ and integrate with respect to $\xi_{1}$ over $\left(\xi_{2}, \infty\right), \xi_{2}>0$. Upon $m$ such integrations we find that

$$
\begin{aligned}
\frac{(-1)^{m}}{2 \pi i} \int_{(\gamma)} & \frac{2^{2 s} \Gamma(s+m) y^{2 r-2 s}}{\Gamma(r+q+m-s)(r+m-s-1) \cdots(r-s)} d s \\
= & 2^{r+q+m-1} \int_{y}^{\infty} \xi_{m-1}^{-3} d \xi_{m-1} \cdots \int_{\xi_{1}}^{\infty} u^{r+2 m-q-2} J_{r+q+2 m-1}(u) d u \\
& =(-1)^{m-1} 2^{r+q} \int_{y}^{\infty} u^{r+2 m-q-2} J_{r+q+2 m-1}(u) r(u, y ; m-1) d u
\end{aligned}
$$

by Lemma 2.1. Let $y=\xi$ and multiply both sides of (4.6) by $x^{q+m-1}$ and differentiate both sides $m-1$ times with respect to $x$ to obtain

$$
\begin{aligned}
\frac{1}{2 \pi i} \int_{(\gamma)} \frac{\Gamma(s+m)\left(\mu_{n} x\right)^{r-s}}{\Gamma(r+q+1-s)(r+m-s-1) \cdots(r-s)} d s \\
\quad=-2^{q-r} x^{-q} \frac{d^{m-1}}{d x^{m-1}}\left(x^{q+m-1} \int_{\xi}^{\infty} u^{r+2 m-q-2} J_{r+q+2 m-1}(u) r(u, \xi ; m-1) d u\right) .
\end{aligned}
$$

Upon substituting the above into (4.3) we arrive at (4.1) provided that

$$
\operatorname{Re} q>\sup (2 \gamma-r, r+2 m-5 / 2) \text {. }
$$


However, the right-hand side of (4.1) converges absolutely and uniformly for $\operatorname{Re} q>\sup \left(2 \sigma_{a}^{*}-r-1 / 2+\delta, r+2 m-5 / 2\right), \delta>0$, by Lemma 2.3. Thus, by analytic continuation in $q,(4.1)$ is valid for $\operatorname{Re} q>\sup \left(2 \sigma_{2}^{*}-r-1 / 2, r+2 m-5 / 2\right)$. If $q$ is real, (4.1) is valid for $q>\sup \left(2 \sigma_{a}^{*}-r-1 / 2, r+2 m-5 / 2\right)$. The convergence is also uniform in $x$ on any interval in $x>0$. This implies that $q>0$ since the left side of (4.1) is not continuous in $x$ for $q \leqq 0$. This completes the proof.

5. The functional equation $\Gamma^{2}(\{s+1\} / 2) \varphi(s)=\Gamma^{2}(\{2-s\} / 2) \psi(1-s)$.

THEOREM 5.1. Let $\varphi$ satisfy the functional equation

$$
\Gamma^{2}(\{s+1\} / 2) \varphi(s)=\Gamma^{2}(\{2-s\} / 2) \psi(1-s)
$$

Suppose that $x>0$ and $q$ is a positive integer.

(i) Let

$$
G_{v}(x)=Y_{v}(x)+e^{\pi i v}\left(2 K_{v}(x) / \pi\right) .
$$

If $q>2 \sigma_{a}^{*}-3 / 2$, then

$$
A_{q}(x)=Q_{q}(x)+2^{-q} \sum b(n)\left(\frac{x}{\mu_{n}}\right)^{(q+1) / 2} G_{q+1}\left(4\left\{\mu_{n} x\right\}^{1 / 2}\right),
$$

where

$$
Q_{q}(x)=\frac{1}{2 \pi i} \int_{C} \frac{\Gamma(s) \varphi(s) x^{s+q}}{\Gamma(s+q+1)} d s
$$

where $C$ is a curve, or curves, encircling all of the integrand's singularities.

(ii) Suppose that $\sup \left(1, \sigma_{a}, \sigma_{a}^{*}\right)<\gamma<7 / 4$. Let

$$
R(x ; q)=\frac{1}{2 \pi i} \int_{C} \frac{\varphi(s) x^{s}}{s^{q+1}} d s
$$

where $C$ is a curve, or curves, encircling the singularities of the integrand in the strip $1-\gamma<\sigma<\gamma$. For $q \geqq 2$,

$$
S(x ; q)=R(x ; q)
$$

$$
+\frac{1}{2} \sum \frac{b(n)}{\mu_{n}} \int_{u_{n} x}^{\infty}\left\{Y_{0}\left(4 u^{1 / 2}\right)-\frac{2}{\pi} K_{0}\left(4 u^{1 / 2}\right)\right\} s\left(u, \mu_{n} x ; q-2\right) d u .
$$

If $\sigma_{a}^{*}<5 / 4$

$$
S(x ; 1)=R(x ; 1)-\frac{1}{2} \sum \frac{b(n)}{\mu_{n}}\left\{Y_{0}\left(4\left\{\mu_{n} x\right\}^{1 / 2}\right)-\frac{2}{\pi} K_{0}\left(4\left\{\mu_{n} x\right\}^{1 / 2}\right)\right\}
$$

(i) is not new and was proved by Chandrasekharan and Narasimhan [7]. Their proof depended upon the evaluation of a certain integral by the use of the residue theorem. However, we shall show below (Lemma 5.3) that the integral can be evaluated very simply by using well-known formulas for Bessel functions. The 
reader may then consult [7] for an indication of the remainder of the proof as well as two extensions of the theorem.

Concerning (ii), again we are not exhaustive in the types of identities we can state here. Removing the restrictions on $\sigma_{a}$ and $\sigma_{a}^{*}$, with the use of Lemmas 5.2 and 5.3, we can derive more complicated identities.

Lemma 5.2. Let $G_{v}$ be defined by (5.1) and let

$$
g_{v}(s, x)=\Gamma(s) \Gamma(s-v)\left\{\frac{-\cos \{\pi(s-v)\}+e^{\pi i v}}{\pi}\right\}\left(\frac{x}{2}\right)^{v-2 s} .
$$

Then, for $0<c<\operatorname{Re} \nu / 2$ and $\operatorname{Re} \nu \neq c+n$, where $n$ is a nonnegative integer,

$$
G_{v}(x)=\frac{1}{2 \pi i} \int_{(c)} g_{v}(s, x) d s-\sum_{k=0}^{[\operatorname{Re} v-c]} \mathscr{R}\left(g_{v}(s, x), \nu-k\right) .
$$

Proof. By (2.13) and (2.14) for $0 \leqq \operatorname{Re} v<\gamma<\operatorname{Re} v / 2+3 / 4<3 / 2$,

$$
G_{v}(x)=\frac{1}{2 \pi i} \int_{(\gamma)} g_{v}(s, x) d s .
$$

We move the line of integration to $\sigma=c, 0<c<\operatorname{Re} \gamma / 2$, by integrating around a rectangle with vertices $\gamma \pm i R$ and $c \pm i R, R>0$. By (2.1) the integrand on $\sigma=c$ is

$$
O\left(|t|^{2 c-\operatorname{Re} v-1}\right)=O(1)
$$

as $|t|$ tends to $\infty$, as $c<\operatorname{Re} \nu / 2$. By a version of the Phragmén-Lindelöf theorem $[15$, p. 109], the integrand is $o(1)$ as $|t|$ tends to $\infty$, uniformly in the strip $c \leqq \sigma \leqq \gamma$. Hence, the integrals over the horizontal sides tend to 0 as $R$ tends to $\infty$. Hence, by the residue theorem,

$$
G_{v}(x)=\frac{1}{2 \pi i} \int_{(c)} g_{v}(s, x) d s+R,
$$

where $R$ is the sum of the residues of $g_{v}(s, x)$ in the strip $c<\sigma<\gamma$. By analytic continuation in $\nu,(5.6)$ may be extended to $\operatorname{Re} v<$ least integer greater than $\gamma$. By repeatedly shifting the line of integration, $\sigma=c$, and using analytic continuation, we eventually arrive at (5.5) provided that $c<\operatorname{Re} \nu / 2$ and $\operatorname{Re} \nu \neq c+n$.

LEMMA 5.3. Let $v$ be a positive integer with $0<c<\nu / 2$, where $\nu-c \neq n$, where $n$ is a positive integer. Then,

$$
\begin{aligned}
\frac{1}{2 \pi i} \int_{(c)} \frac{\Gamma(1-s) \Gamma^{2}(\{s+1\} / 2)}{\Gamma(\nu+1-s) \Gamma^{2}(\{2-s\} / 2)} & \left(\frac{x}{2}\right)^{v-2 s} d s \\
& =2^{-v+1}\left\{G_{v}(2 x)+\sum_{k=0}^{[v-c]} \mathscr{R}\left(g_{v}(s, 2 x), v-k\right)\right\} .
\end{aligned}
$$


Proof. By (2.3), three applications of (2.2), and elementary trigonometry we have

$$
\begin{aligned}
\frac{\Gamma(1-s) \Gamma^{2}(\{s+1\} / 2)}{\Gamma(\nu+1-s) \Gamma^{2}(\{2-s\} / 2)} & =\frac{2^{2-2 s} \pi \Gamma(1-s) \Gamma^{2}(s)}{\Gamma(\nu+1-s) \Gamma^{2}(\{2-s\} / 2) \Gamma^{2}(s / 2)} \\
& =\frac{2^{2-2 s} \Gamma(1-s) \Gamma^{2}(s) \sin ^{2}(\pi s / 2)}{\pi \Gamma(\nu+1-s)} \\
& =\frac{\Gamma(s-\nu) \sin \{\pi(s-\nu)\} \Gamma(s)(1-\cos (\pi s))}{\pi 2^{2 s-1} \sin (\pi s)} \\
& =\frac{\Gamma(s-\nu) \Gamma(s)\left\{(-1)^{\nu}-\cos (\pi(s-\nu))\right\}}{\pi 2^{2 s-1}} .
\end{aligned}
$$

Hence,

$$
\frac{1}{2 \pi i} \int_{(c)} \frac{\Gamma(1-s) \Gamma^{2}(\{s+1\} / 2)}{\Gamma(\nu+1-s) \Gamma^{2}(\{2-s\} / 2)}\left(\frac{x}{2}\right)^{\nu-2 s} d s=\frac{2^{-v+1}}{2 \pi i} \int_{(c)} g_{v}(s, 2 x) d s,
$$

and the proof is complete by Lemma 5.2.

Proof of (ii). Proceeding as in [2, proof of Theorem 1], we arrive at

$$
S(x ; q)=R(x ; q)+\sum \frac{b(n)}{\mu_{n}} \frac{1}{2 \pi i} \int_{(\gamma)} \frac{\Gamma^{2}(\{s+1\} / 2)\left(\mu_{n} x\right)^{1-s}}{\Gamma^{2}(\{2-s\} / 2)(1-s)^{q+1}} d s,
$$

provided that $q>2 \gamma-1$.

Assume first that $q \geqq 2$. Then, by (2.4),

$$
\begin{aligned}
\frac{\Gamma^{2}(\{s+1\} / 2)}{\Gamma^{2}(\{2-s\} / 2)(1-s)^{2}} & =\frac{\Gamma^{2}(\{s-1\} / 2)}{4 \Gamma^{2}(\{2-s\} / 2)} \\
& =\frac{\Gamma^{2}(s-1)\{1+\cos (\pi(s-1))\}}{\pi 2^{2 s-1}} .
\end{aligned}
$$

It follows by (2.13) and (2.14) that for $1<\gamma<7 / 4$,

$$
\frac{1}{2 \pi i} \int_{(\gamma)} \frac{\Gamma^{2}(\{s+1\} / 2) u^{-s}}{\Gamma^{2}(\{2-s\} / 2)(1-s)^{2}} d s=-\frac{1}{2 u}\left\{Y_{0}\left(4 u^{1 / 2}\right)-\frac{2}{\pi} K_{0}\left(4 u^{1 / 2}\right)\right\}
$$

Integrate both sides of (5.8) with respect to $u$ over $\left(\xi_{1}, \infty\right), \xi_{1}>0$, and proceed exactly as in the proof of Theorem 3.1, (ii). After $q-1$ integrations, we find by (2.16), (2.17) and Lemma 2.2 that

$$
\begin{aligned}
& \frac{1}{2 \pi i} \int_{(\gamma)} \frac{\Gamma^{2}(\{s+1\} / 2) y^{1-s}}{\Gamma^{2}(\{2-s\} / 2)(1-s)^{q+1}} d s \\
&=\frac{1}{2} \int_{y}^{\infty} \frac{1}{u}\left\{Y_{0}\left(4 u^{1 / 2}\right)-\frac{2}{\pi} K_{0}\left(4 u^{1 / 2}\right)\right\} s(u, y ; q-2) d u .
\end{aligned}
$$

Setting $y=\mu_{n} x$ and substituting into (5.7), we have established (5.3) provided that $q>2 \gamma-1$. However, by (2.16), (2.17) and Lemma 2.3, the right-hand side of (5.9) is

$$
O\left(\left\{\mu_{n} x\right\}^{-3 / 2} \log ^{q-2} \mu_{n} x\right) .
$$


Since $\sigma_{a}^{*}<7 / 4$, the right-hand side of (5.3) converges absolutely and uniformly in $x$ on any interval in $x>0$. Hence, by (2.24) and (2.25), (5.3) may be upheld for $q \geqq 2$ by differentiation with respect to $x$.

(5.4) may be established by putting $q=2$ in (5.3) and then differentiating with respect to $x$. By $(2.16)$ and $(2.17)$ the differentiation is justified provided that $\sigma_{a}^{*}<5 / 4$.

6. $\Gamma(s / 2) \Gamma(\{s-p\} / 2) \varphi(s)=\Gamma(\{1-s\} / 2) \Gamma(\{p+1-s\} / 2) \psi(p+1-s)$, the functional equation.

THEOREM 6.1. Let $\varphi$ satisfy the functional equation

$$
\Gamma(s / 2) \Gamma(\{s-p\} / 2) \varphi(s)=\Gamma(\{1-s\} / 2) \Gamma(\{p+1-s\} / 2) \psi(p+1-s),
$$

where $p$ is an integer. Let $x>0, \xi=2\left(\mu_{n} x\right)^{1 / 2}$, and suppose that $q$ is a positive integer. Define

$$
F_{v, p}(x)=\cos (\{p+1\} \pi / 2) J_{p+v}(x)-\sin (\{p+1\} \pi / 2) F_{p+v}(x),
$$

where $F_{v}(x)=Y_{v}(x)-e^{\pi i v}\left(2 K_{v}(x) / \pi\right)$.

(i) For $q>2 \sigma_{a}^{*}-p-3 / 2$,

$$
A_{q}(x)=Q_{q}(x)+2^{-q} \sum b(n)\left(\frac{x}{\mu_{n}}\right)^{(q+p+1) / 2} F_{q+1, p}(2 \xi),
$$

where

$$
Q_{q}(x)=\frac{1}{2 \pi i} \int_{C} \frac{\Gamma(s) \varphi(s) x^{s+q}}{\Gamma(s+q+1)} d s,
$$

where $C$ is a curve, or curves, encircling all of the integrand's singularities.

(ii) If $q>\sup \left(2 \sigma_{a}^{*}-p-3 / 2, p+1 / 2\right)$, then

$$
\begin{aligned}
S(x ; q)= & R(x ; q)+2^{q-p-1} \sum \frac{b(n)}{\mu_{n}^{p+1}} \frac{d^{q-1}}{d x^{q-1}} \\
& \cdot\left(x^{q-1} \int_{\xi}^{\infty} u^{p+1-q} F_{q, p}(2 u) s(u, \xi ; q-1) d u\right),
\end{aligned}
$$

where

$$
R(x ; q)=\frac{1}{2 \pi i} \int_{C} \frac{\varphi(s) x^{s}}{s^{q+1}} d s,
$$

where $C$ is a curve, or curves, encircling all of the integrand's singularities.

(i) is due to Chandrasekharan and Narasimhan [7]. Their proof depended upon the evaluation of a certain integral. However, again this integral can be evaluated simply from well-known Bessel function formulas as we indicate below. The reader may then consult [7] for the remainder of the proof as well as two extensions of the theorem.

In (ii), if we require that $\sigma_{a}, \sigma_{a}^{*}<p+1$, we can derive an identity for $2 \sigma_{a}^{*}-p-3 / 2$ $<q \leqq p+1 / 2$. The proof follows along the same lines as that in [2, Theorem 3]. 
Using the method of equiconvergent trigonometric series, we can extend our results to $q>2 \sigma_{a}^{*}-p-5 / 2, q \geqq 0$.

LEMMA 6.2. Let $F_{v, p}$ be as given in (6.1). Let $\nu$ be a positive integer and $0<c$ $<(p+\nu) / 2$, where $p$ is an integer and $p+\nu-c \neq n$, where $n$ is a nonnegative integer. Then,

$$
\begin{aligned}
I & =\frac{1}{2 \pi i} \int_{(c)} \frac{\Gamma(p+1-s) \Gamma(s / 2) \Gamma(\{s-p\} / 2)}{\Gamma(p+1+\nu-s) \Gamma(\{1-s\} / 2) \Gamma(\{p+1-s\} / 2)}\left(\frac{x}{2}\right)^{p+\nu-2 s} d s \\
& =2^{-v+1}\left\{F_{v, p}(2 x)+\sum_{k=0}^{[p+v-c]} \mathscr{R}\left(f_{v, p}(s, 2 x), p+\nu-k\right)\right\},
\end{aligned}
$$

where

$$
f_{v, p}(s, x)=\sin (\{p+1\} \pi / 2) \Gamma(s) \Gamma(s-p-\nu)\left\{\frac{\cos \{\pi(s-p-\nu)\}+e^{\pi i(p+\nu)}}{\pi}\right\}\left(\frac{x}{2}\right)^{p+\nu-2 s} .
$$

Proof. By the same method as in the proof of Lemma 5.2, for $\nu$ complex

$$
\begin{aligned}
& -\sin (\{p+1\} \pi / 2) F_{p+v}(x) \\
& =\frac{1}{2 \pi i} \int_{(c)} f_{v, p}(s, x) d s-\sum_{k=0}^{[p+\operatorname{Re} v-c]} \mathscr{R}\left(f_{v, p}(s, x), p+\nu-k\right),
\end{aligned}
$$

where $0<c<(p+\operatorname{Re} v) / 2$ and $p+\operatorname{Re} \nu \neq c+n$.

By two applications of both (2.4) and (2.2),

$$
\begin{aligned}
\frac{\Gamma(p+1-s) \Gamma(s / 2) \Gamma(\{s-p\} / 2)}{\Gamma(p+1+\nu-s) \Gamma(\{1-s\} / 2) \Gamma(\{p+1-s\} / 2)} \\
=\frac{\cos (\pi s / 2) \cos (\pi(s-p) / 2) \Gamma(s) \Gamma(s-p) \Gamma(p+1-s)}{\pi 2^{2 s-p-2} \Gamma(p+1+\nu-s)} \\
=\frac{\{(1+\cos (\pi s)) \cos (\pi p / 2)+\sin (\pi s) \sin (\pi p / 2)\} \Gamma(s)}{2^{2 s-p-1} \Gamma(p+1+\nu-s) \sin \{\pi(s-p)\}} \\
=\frac{\sin (\pi s) \sin (\pi p / 2) \Gamma(s)}{2^{2 s-p-1} \Gamma(p+1+\nu-s) \sin \{\pi(s-p)\}} \\
\quad+\frac{(1+\cos (\pi s)) \cos (\pi p / 2) \Gamma(s) \Gamma(s-p-\nu) \sin \{\pi(s-p-\nu)\}}{\pi 2^{2 s-p-1} \sin \{\pi(s-p)\}} .
\end{aligned}
$$

Whether $p$ is even or odd, the first term on the right-hand side of (6.5) may be written as

$$
\frac{\cos (\{p+1\} \pi / 2) \Gamma(s)}{2^{2 s-p-1} \Gamma(p+1+\nu-s)}
$$

Since $v$ is integral, the second term on the right-hand side of (6.5) may be written as

$$
\begin{aligned}
& \frac{\left\{(-1)^{\nu}+\cos (\pi(s-\nu))\right\} \cos (\pi p / 2) \Gamma(s) \Gamma(s-p-\nu)}{\pi 2^{2 s-p-1}} \\
& =\frac{\left\{(-1)^{\nu+p}+\cos (\pi(s-p-\nu))\right\} \sin (\{p+1\} \pi / 2) \Gamma(s) \Gamma(s-p-\nu)}{\pi 2^{2 s-p-1}}
\end{aligned}
$$


for $p$ either even or odd. Hence,

$$
I=\frac{2^{-\nu+1}}{2 \pi i} \int_{(c)}\left\{\frac{\cos (\{p+1\} \pi / 2) \Gamma(s)}{\Gamma(p+1+\nu-s)}+f_{v, p}(s, 2 x)\right\} x^{-2 s} d s,
$$

and the proof is complete by (2.12) and (6.4).

Proof of Theorem 6.1, (ii). Proceeding as in [2, proof of Theorem 1], we find that

$$
\begin{aligned}
S(x ; q)= & R(x ; q)+\sum \frac{b(n)}{\mu_{n}^{p+1}} \frac{1}{2 \pi i} \\
& \cdot \int_{(\gamma)} \frac{\Gamma(s / 2) \Gamma(\{s-p\} / 2)\left(\mu_{n} x\right)^{p+1-s}}{\Gamma(\{1-s\} / 2) \Gamma(\{p+1-s\} / 2)(p+1-s)^{q+1}} d s,
\end{aligned}
$$

where $\gamma$ is such that $\gamma>\sup \left(0, \sigma_{a}, \sigma_{a}^{*}, p+1\right)$ and $S$ lies in the strip $p+1-\gamma<\sigma<\gamma$, and where $q>2 \gamma-p-1$.

Assume first that $p$ and $q$ are both even. Then, by (2.4),

$$
\begin{aligned}
h_{q, p}(s) & =\frac{\Gamma(s / 2) \Gamma(\{s-p-q\} / 2)}{\Gamma(\{1-s\} / 2) \Gamma(\{p+q+1-s\} / 2)} \\
& =\pi^{-1} 2^{p+q+2-2 s} \cos (\pi s / 2) \cos (\pi(s-p-q) / 2) \Gamma(s) \Gamma(s-p-q) \\
& =(-1)^{(p+q) / 2} \pi^{-1} 2^{p+q+1-2 s}\{1+\cos (\pi(s-p-q))\} \Gamma(s) \Gamma(s-p-q) .
\end{aligned}
$$

If $p$ is odd and $q$ is even, by (2.4), (2.3) and (2.2),

$$
\begin{aligned}
h_{q, p}(s) & =\frac{2^{p+q+1-2 s} \cos (\pi s / 2) \Gamma(s) \Gamma(\{s-p-q\} / 2) \Gamma(1+\{p+q-s\} / 2)}{\pi \Gamma(p+q+1-s)} \\
& =\frac{2^{p+q+1-2 s} \cos (\pi s / 2) \Gamma(s)}{\Gamma(p+q+1-s) \sin (\pi(s-p-q) / 2)} \\
& =\frac{(-1)^{(p+q+1) / 2} 2^{p+q+1-2 s} \Gamma(s) .}{\Gamma(p+q+1-s)} .
\end{aligned}
$$

It then follows from (6.4) that for $0<c<(p+q) / 2, q$ even, and $p$ odd or even that

or

$$
\begin{aligned}
\frac{1}{2 \pi i} \int_{(\gamma)} h_{q, p}(s)\left(\frac{u}{2}\right)^{p+q-2 s} d s & \\
& =2(-1)^{q / 2}\left\{-F_{q, p}(2 u)+\sum_{k=0}^{[p+q-\gamma]} \mathscr{R}\left(f_{q, p}(s, 2 u), p+q-k\right)\right\},
\end{aligned}
$$

$$
\frac{1}{2 \pi i}\left(\int_{(\gamma)}-\int_{C_{p+q}}\right) h_{q, p}(s)\left(\frac{u}{2}\right)^{p+q-2 s} d s=2(-1)^{(q+2) / 2} F_{q, p}(2 u),
$$

where $C_{p+q}$ is a curve, or curves, encircling the singularities of the integrand in the strip $\gamma<\sigma<(p+q) / 2$.

Now, multiply both sides of (6.7) by $u^{p-q+1}$ and integrate over $\left(\xi_{1}, \infty\right), \xi_{1}>0$. Since $\gamma>p+1$, the resulting integral on the left side converges. By (2.15)-(2.17) 
the integral on the right side is convergent provided that $q>p+1 / 2$. If $q>2 \gamma-p-1$ we may invert the order of integration by $[13$, p. 349]. We therefore obtain

$$
\begin{aligned}
&-\frac{1}{2 \pi i}\left(\int_{(\gamma)}-\int_{C_{p+q}}\right) \frac{h_{q, p}(s) 2^{2 s-p-q-1} \xi_{1}^{2 p+2-2 s}}{p+1-s} d s \\
&=2(-1)^{(q+2) / 2} \int_{\xi_{1}}^{\infty} u^{p-q+1} F_{q, p}(2 u) d u .
\end{aligned}
$$

Proceeding exactly as in [2, proof of Theorem 1, (ii)], we find after $q$ such integrations and an application of Lemma 2.2 that

$$
\begin{aligned}
\frac{1}{2 \pi i}\left(\int_{(\gamma)}-\int_{C_{p+q}}\right) \frac{h_{q, p}(s) 2^{2 s-p-2 q} y^{2 p+2-2 s}}{(p+1-s)^{q}} d s \\
=2(-1)^{q / 2} \int_{y}^{\infty} u^{p-q+1} F_{q, p}(2 u) s(u, y ; q-1) d u .
\end{aligned}
$$

Let $y=\xi$, multiply both sides of (6.8) by $x^{q-1}$, and differentiate both sides $q-1$ times with respect to $x$. The resulting differentiations under the integral sign on the left are justified by a standard theorem [17, p. 59] for $q>2 \gamma-p-1$. With the help of (2.4) we arrive at

$$
\begin{aligned}
& \frac{(-1)^{q / 2}}{2 \pi i}\left(\int_{(\gamma)}-\int_{C_{p+q}}\right) \frac{h_{0, p}(s) 2^{p-q+2}\left(\mu_{n} x\right)^{p+1-s}}{(p+1-s)^{q+1}} d s \\
& \quad=2(-1)^{q / 2} \frac{d^{q-1}}{d x^{q-1}}\left(x^{q-1} \int_{\xi}^{\infty} u^{p-q+1} F_{q, p}(2 u) s(u, \xi ; q-1) d u\right) .
\end{aligned}
$$

We now substitute (6.9) into (6.6) and note that

$$
\begin{aligned}
& \sum \frac{b(n)}{\mu_{n}^{p+1}} \int_{C_{p+q}} \frac{h_{0, p}(s)\left(\mu_{n} x\right)^{p+1-s}}{(p+1-s)^{q+1}} d s \\
&=\int_{C_{p+q}} \frac{h_{0, p}(s) \psi(s) x^{p+1-s}}{(p+1-s)^{q+1}} d s=\int_{C_{p+q}^{\prime}} \frac{\varphi(s) x^{s}}{s^{q+1}} d s=0,
\end{aligned}
$$

where $C_{p+q}^{\prime}$ is a positively oriented curve, or curves, encircling the singularities of the integrand for $1-q<\sigma<p+1-\gamma$, and there are no such singularities by our choice of $\gamma$. Hence, we have established (6.3) provided that $q$ is even and that $q>\sup (2 \gamma-p-1, p+1 / 2)$.

However, by (2.24), (2.25) and Lemma 2.3, the right side of (6.9) is

$$
\int_{\xi}^{\infty} u^{p-q+1} F_{q, p}(2 u) \sum_{k=0}^{q-1} c_{k, q-1} s(u, \xi ; k) d u=O\left(\xi^{p-q+1 / 2} \log ^{q-1} \xi\right) .
$$

It follows that the series on the right-hand side of (6.3) converges absolutely and uniformly on any interval in $x>0$ provided that $q>2 \sigma_{a}^{*}-p-3 / 2$. We show that (6.3) may be upheld for integral $q>\sup \left(2 \sigma_{a}^{*}-p-3 / 2, p+1 / 2\right)$ by differentiation. In [2, proof of Theorem 1, (ii)] we omitted the verification, which is by no means trivial. We give the details for our theorem here; the details in [2] are similar. 
Let $Z_{n}=\{1,2, \ldots, n\}$. After some calculation, it is not difficult to see that the $c_{k, q-1}$ in $(6.10)$ are given by

$$
c_{k, q-1}=2^{k-q+1} \sum_{i, \in Z_{q-1}} i_{1} i_{2} \ldots i_{k},
$$

where $i_{j} \neq i_{l}$ if $j \neq l$. Thus, the sum is over all products of $k$ distinct integers chosen from $Z_{q-1}$. In particular, $c_{q-1, q-1}=(q-1)$ ! and $c_{0, q-1}=2^{-q+1}$.

We next put $u=\xi v$ in the integral on the left-hand side of (6.10). This integral then becomes with $s(v, 1 ; k)=s(v ; k)$

$$
I_{q}(x)=\xi^{p-q+2} \int_{1}^{\infty} v^{p-q+1} F_{q, p}(2 \xi v) \sum_{k=0}^{q-1} c_{k, q-1} s(v ; k) d v .
$$

From (6.3) we see that we must show that

$$
I_{q}^{\prime}(x)=I_{q-1}(x) / 2 x .
$$

However, since $q>p+1 / 2$, differentiation under the integral sign in (6.11) is generally not permitted. Hence, we first perform an integration by parts. From (2.18)-(2.20) and the definition of $F_{v}$ we find that

and

$$
d\left\{x^{v+p} F_{v, p}(c x)\right\} / d x=c x^{v+p} F_{v-1, p}(c x)
$$

$$
d\left\{x^{(v+p) / 2} F_{v, p}(2 \xi v)\right\} / d x=\xi v x^{(v+p) / 2-1} F_{v-1, p}(2 \xi v) .
$$

Integrating by parts, we have

$$
\begin{aligned}
I_{q}(x)=\frac{1}{2} \xi^{p-q+1} & \left(-2^{1-q} F_{q+1, p}(2 \xi)\right. \\
& \left.-\int_{1}^{\infty} v^{p+q+1} F_{q+1, p}(2 \xi v) \frac{d}{d v}\left\{v^{-2 q} \sum_{k=0}^{q-1} c_{k, q-1} s(v ; k)\right\} d v\right) .
\end{aligned}
$$

Thus,

$$
\begin{aligned}
I_{q}^{\prime}(x)= & \frac{1}{2}\left(2 \mu_{n}^{1 / 2}\right)^{p-q+1} q x^{-q-1} \\
& \times\left(2^{1-q} x^{(p+q+1) / 2} F_{q+1, p}(2 \xi)+x^{(p+q+1) / 2} \int_{1}^{\infty} v^{p+q+1} F_{q+1, p}(2 \xi v) \frac{d}{d v}\right. \\
& \left.\times\left\{v^{-2 q} \sum_{k=0}^{q-1} c_{k, q-1} s(v ; k)\right\} d v\right) \\
& -\frac{1}{2}\left(2 \mu_{n}^{1 / 2}\right)^{p-q+2} x^{-q} \\
& \times\left(2^{1-q} x^{(p+q) / 2} F_{q, p}(2 \xi)+x^{(p+q) / 2} \int_{1}^{\infty} v^{p+q+2} F_{q, p}(2 \xi v) \frac{d}{d v}\right. \\
& \left.\times\left\{v^{-2 q} \sum_{k=0}^{q-1} c_{k, q-1} s(v ; k)\right\} d v\right) .
\end{aligned}
$$

Note that $1 / 2 x$ times the first expression on the right-hand side of (6.13) with $q$ replaced by $q-1$ is equal to the third expression on the right-hand side of (6.14). 
Upon an integration by parts, the first two expressions on the right-hand side of (6.14) yield

$$
-\xi^{p-q+2} q x^{-1} \int_{1}^{\infty} v^{p-q+1} F_{q, p}(2 \xi v) \sum_{k=0}^{q-1} c_{k, q-1} s(v ; k) d v
$$

Thus, from (6.13), (6.14) and the above we deduce that we must show that

$$
\begin{aligned}
q v^{-2 q+1} \sum_{k=0}^{q-1} c_{k, q-1} s(v ; k)+\frac{1}{2} v^{2} \frac{d}{d v}\left\{v^{-2 q}\right. & \left.\sum_{k=0}^{q-1} c_{k, q-1} s(v ; k)\right\} \\
& =\frac{1}{4} \frac{d}{d v}\left\{v^{-2 q+2} \sum_{k=0}^{q-2} c_{k, q-2} s(v ; k)\right\} .
\end{aligned}
$$

Upon differentiation and simplification, the above reduces to

$$
\begin{aligned}
& -\frac{v^{-2 q+1}}{2} \sum_{k=0}^{q-2} c_{k+1, q-1} s(v ; k) \\
& =\frac{v^{-2 q+1}}{4}\left\{(-2 q+2) \sum_{k=0}^{q-2} c_{k, q-2} s(v ; k)-\sum_{k=0}^{q-3} c_{k+1, q-2} s(v ; k)\right\} .
\end{aligned}
$$

Hence, we must show that

$$
\begin{aligned}
c_{k+1, q-1} & =(q-1) c_{k, q-2}+\frac{1}{2} c_{k+1, q-2}, & & k=0,1, \ldots, q-3, \\
c_{k+1, q-1} & =(q-1) c_{k, q-2}, & k & =q-2 .
\end{aligned}
$$

The last relation is obvious. Now,

$$
\begin{aligned}
(q-1) c_{k, q-2}+ & \frac{1}{2} c_{k+1, q-2} \\
& =(q-1) 2^{k-q+2} \sum_{i_{j} \in Z_{q}-2} i_{1} i_{2} \ldots i_{k}+2^{k-q+2} \sum_{i_{j} \in Z_{q-2}} i_{1} i_{2} \ldots i_{k+1} \\
& =2^{k-q+2} \sum_{i_{j} \in Z_{q-1}} i_{1} i_{2} \ldots i_{k+1}=c_{k+1, q-1} .
\end{aligned}
$$

This completes the proof of (6.12) and also the proof of the theorem.

7. An identity involving $\mathrm{Ei}(-x)$.

THEOREM 7.1. For $x>0$ let

$$
\tilde{\Phi}(x)=\sum a(n) \mathrm{Ei}\left(-\lambda_{n} x\right), \quad \tilde{\Psi}(x)=\sum b(n) \mathrm{Ei}\left(-\mu_{n} \cdot x\right),
$$

where $\mathrm{Ei}(-x)$ is defined by (2.7). Then $\varphi$ satisfies functional equation (1.3) if and only if

$$
\tilde{\Phi}(x)=-R(x)+x^{-r} \tilde{\Psi}(1 / x),
$$

where $R(x)$ is a residual function (see [1], [4] or [5]) given by

$$
R(x)=\frac{1}{2 \pi i} \int_{C} \frac{\Gamma(s) \varphi(s) x^{-s}}{s} d s,
$$

where $C$ is a curve, or curves, encircling the singularities of the integrand. 
Proof. The proof closely resembles that in [1, Theorem 1], and so we give just a brief sketch. Choose $\gamma$ so that $\gamma>\sup \left(0, \sigma_{a}, \sigma_{a}^{*}\right)$ and such that $S$ lies in the strip $r-\gamma<\sigma<\gamma$. From (2.9) it follows easily that

$$
-\frac{1}{2 \pi i} \int_{(y)} \frac{\Gamma(s) \varphi(s) x^{-s}}{s} d s=\tilde{\Phi}(x) .
$$

On the other hand,

$$
\begin{aligned}
-\frac{1}{2 \pi i} \int_{(\gamma)} \frac{\Gamma(s) \varphi(s) x^{-s}}{s} d s & =-R(x)-\frac{1}{2 \pi i} \int_{(r-\gamma)} \frac{\Gamma(s) \varphi(s) x^{-s}}{s} d s \\
& =-R(x)+x^{-r \tilde{\Psi}(1 / x),}
\end{aligned}
$$

upon using the functional equation, replacing $s$ by $r-s$, and inverting the order of summation and integration.

To prove the converse we employ (2.8) and find that for $\sigma>\sigma_{a}$

$$
\Gamma(s) \varphi(s)=-s \int_{0}^{\infty} x^{s-1} \tilde{\Phi}(x) d x .
$$

The remainder of the proof parallels that in [1, Theorem 1].

\section{Generalized Dirichlet series.}

THEOREM 8.1. Let $\varphi$ satisfy (1.3) and define for $a>0$ and $\sigma>\sigma_{a}$,

$$
\varphi(s, a)=\sum a(n)\left(a+\lambda_{n}\right)^{-s} .
$$

Let $\xi=2\left(\mu_{n} a\right)^{1 / 2}$ and let $\mathscr{D}$ be a domain where

$$
\sum b(n) K_{s-r}(\xi) \mu_{n}^{(s-r) / 2}
$$

converges uniformly. Suppose that the singularities of $\chi$ are at most poles. Let $R(s, a)$ denote the sum of the residues of $\chi(w) \Gamma(s-w) a^{w-s}$ at the poles of $\chi(w)$. Then, if $s \in \mathscr{D}$,

$$
\Gamma(s) \varphi(s, a)=2 \sum b(n)\left(\frac{\mu_{n}}{a}\right)^{(s-r) / 2} K_{s-r}(\xi)+R(s, a) .
$$

Conversely, if $\varphi(s, a)$ satisfies (8.1), then $\varphi$ satisfies (1.3).

Proof. For $\sigma>\sup \left(0, \sigma_{a}, \sigma_{a}^{*}\right)$,

$$
\begin{aligned}
\Gamma(s) \varphi(s, a) & =\sum a(n) \int_{0}^{\infty} y^{s-1} \exp \left(-\left(a+\lambda_{n}\right) y\right) d y \\
& =\int_{0}^{\infty} y^{s-1} e^{-a y} \Phi(y) d y \\
& =\int_{0}^{\infty} y^{s-1} e^{-a y} P(y) d y+\sum b(n) \int_{0}^{\infty} y^{s-r-1} \exp \left(-a y-\mu_{n} / y\right) d y
\end{aligned}
$$

by (1.4), where all inversions in order of summation and integration are justified 
by absolute convergence. Using the definition of $P(y)$ and inverting the order of integration, we find that

$$
\int_{0}^{\infty} y^{s-1} e^{-a y} P(y) d y=\frac{1}{2 \pi i} \int_{C} \chi(w) \Gamma(s-w) a^{w-s} d w,
$$

provided that $\operatorname{Re}(s-w)>0$. But this is a priori satisfied since $\sigma>\sigma_{a}>\operatorname{Re} w$. Using (2.10), we have

$$
\sum b(n) \int_{0}^{\infty} y^{s-r-1} \exp \left(-a y-\mu_{n} / y\right) d y=2 \sum b(n)\left(\frac{\mu_{n}}{a}\right)^{(s-r) / 2} K_{s-r}(\xi) .
$$

Combining (8.2)-(8.4), we have established (8.1) for $\sigma>\sup \left(0, \sigma_{a}, \sigma_{a}^{*}\right)$. However, by analytic continuation (8.1) is valid for the indicated values of $s$.

To prove the converse, we multiply both sides of $(8.1)$ by $y^{-s} e^{a y} / 2 \pi i$ and integrate with respect to $s$ over $(c-i \infty, c+i \infty)$ with $c>\sup \left(0, \sigma_{a}, \sigma_{a}^{*}\right)$.

First, by absolute convergence and (2.5)

$$
\begin{aligned}
\frac{1}{2 \pi i} \int_{(c)} y^{-s} e^{a y} \Gamma(s) \varphi(s, a) d s & =\sum a(n) e^{a y} \frac{1}{2 \pi i} \int_{(c)} \Gamma(s)\left\{\left(a+\lambda_{n}\right) y\right\}^{-s} d s \\
& =\Phi(y) .
\end{aligned}
$$

Secondly, by using the integral representation for $R(s, a)$, inverting the order of integration, and employing (2.5), we easily deduce that

$$
\frac{1}{2 \pi i} e^{a y} \int_{(c)} R(s, a) y^{-s} d s=P(y)
$$

Thirdly, by inverting the order of summation and integration by uniform convergence and using (2.11), we have

$$
\begin{aligned}
\frac{1}{2 \pi i} e^{a y} \int_{(c)} y^{-s} 2 \sum b(n)\left(\frac{\mu_{n}}{a}\right)^{(s-r) / 2} & K_{s-r}(\xi) d s \\
& =2 e^{a y} y^{-r} \sum b(n) \int_{(c-r)} K_{s}(\xi)\left(\frac{a y^{2}}{\mu_{n}}\right)^{-s / 2} d s \\
& =y^{-r} \Psi(1 / y) .
\end{aligned}
$$

Combining (8.5)-(8.7) we arrive at (1.4), and the proof is complete.

9. An identity involving $K_{v}(x)$.

THEOREM 9.1. If $\varphi$ satisfies functional equation (1.3), then

$$
\begin{aligned}
2 \sum a(n)\left(\frac{b}{\lambda_{n}+a}\right)^{v / 2} K_{v}\left(2\left\{\left(\lambda_{n}+a\right) b\right\}^{1 / 2}\right) \\
\quad=\int_{0}^{\infty} x^{\nu-1} e^{-a x-b / x} P(x) d x+2 \sum b(n)\left(\frac{a}{\mu_{n}+b}\right)^{(r-v) / 2} K_{r-v}\left(2\left\{\left(\mu_{n}+b\right) a\right\}^{1 / 2}\right),
\end{aligned}
$$

where $\operatorname{Re} a, \operatorname{Re} b>0$ and $\nu$ is an arbitrary complex number. 
Dixon and Ferrar [9] have proven (9.1) in a more complicated way for the special case $\varphi(s)=(2 \pi)^{-s} \sum r_{2}(n) n^{-s}$, where $r_{2}(n)$ is the number of representations of $n$ as the sum of 2 squares.

Proof. From (2.10) and (1.4) we have

$$
\begin{aligned}
\left.2 \sum a(n)\left(\frac{b}{\lambda_{n}+a}\right)^{v / 2} K_{v}\left(2\left\{\lambda_{n}+a\right) b\right\}^{1 / 2}\right) \\
\quad=\sum a(n) \int_{0}^{\infty} x^{\nu-1} \exp \left(-\left(\lambda_{n}+a\right) x-b / x\right) d x \\
\quad=\int_{0}^{\infty} x^{\nu-1} e^{-a x-b / x} \Phi(x) d x \\
\quad=\int_{0}^{\infty} x^{\nu-1} e^{-a x-b / x}\left\{P(x)+x^{-r \Psi}(1 / x)\right\} d x \\
\quad=\int_{0}^{\infty} x^{\nu-1} e^{-a x-b / x} P(x) d x+\sum b(n) \int_{0}^{\infty} x^{r-v-1} \exp \left(-\left(b+\mu_{n}\right) x-a / x\right) d x \\
\quad=\int_{0}^{\infty} x^{\nu-1} e^{-a x-b / x} P(x) d x+2 \sum b(n)\left(\frac{a}{\mu_{n}+b}\right)^{(r-v) / 2} K_{r-v}\left(2\left\{\left(\mu_{n}+b\right) a\right\}^{1 / 2}\right),
\end{aligned}
$$

where all inversions in order of summation and integration are justified by absolute convergence.

CoROllaRY 9.2. If $\nu=1 / 2$ in Theorem 9.1, we have

$$
\begin{aligned}
\pi^{1 / 2} \sum a(n)\left(\lambda_{n}+a\right)^{-1 / 2} \exp \left(-2\left\{\left(\lambda_{n}+a\right) b\right\}^{1 / 2}\right) \\
\quad=\int_{0}^{\infty} x^{-1 / 2} e^{-a x-b / x} P(x) d x+2 \sum b(n)\left(\frac{a}{\mu_{n}+b}\right)^{(2 r-1) / 4} K_{r-1 / 2}\left(2\left\{\left(\mu_{n}+b\right) a\right\}^{1 / 2}\right) .
\end{aligned}
$$

Proof. Use (2.21), and the result follows.

\section{Identities involving Laguerre polynomials.}

THEOREM 10.1. Let $y>0, m$ be a positive integer, and $L_{m}^{(\alpha)}(y)$ be a Laguerre polynomial. Then, if $\varphi$ satisfies (1.3),

$$
\begin{aligned}
\sum a(n) \lambda_{n}^{m} \exp \left(-\lambda_{n} y\right)= & m ! y^{-r-m} \sum b(n) \exp \left(-\mu_{n} / y\right) L_{m}^{(r-1)}\left(\mu_{n} / y\right) \\
& +\frac{1}{2 \pi i} \int_{C} \frac{\chi(s) \Gamma(s+m) y^{-s-m}}{\Gamma(s)} d s
\end{aligned}
$$

where $C$ is a curve, or curves, encircling the singularities of $\chi$, and where if $s \in C$, $s+n \neq 0, n=1,2, \ldots, m$.

THEOREM 10.2. Let $y>0, q \geqq 0, m$ be a positive integer, and

$$
Q_{q}(x)=\frac{1}{2 \pi i} \int_{C} \frac{\chi(s) x^{s+q}}{\Gamma(s+q+1)} d s,
$$


where $\varphi$ satisfies (1.3) and $C$ is as in Theorem 10.1. Suppose $m$ is large enough so that if $s \in C, \operatorname{Re} s+m+q+1>0$. Also, suppose $s+q+n \neq 0, n=0,1, \ldots, m-1$. Then,

$$
\begin{aligned}
\int_{0}^{\infty} e^{-y x} x^{m}\left\{A_{q}(x)-Q_{q}(x)\right\} d x & \\
& =m ! y^{-m-r-q-1} \sum b(n) \exp \left(-\mu_{n} / y\right) L_{m}^{(r+q)}\left(\mu_{n} / y\right) .
\end{aligned}
$$

These theorems generalize results of Koshliakov [14] and Szegö [16]. An identity for an integral similar to that of (10.2) has been given by Chandrasekharan and Narasimhan [6].

Proof of Theorem 10.1. Write (1.4) in the form

$$
\Phi(1 / y)=y^{r} \Psi(y)+P(1 / y) .
$$

Multiply both sides of (10.3) by $y^{m-1}$ and differentiate $m$ times with respect to $y$. Using (2.22) and the fact that

$$
d^{m}\left(y^{m-1} \exp \left(-\lambda_{n} / y\right)\right) / d y^{m}=\lambda_{n}^{m} y^{-m-1} \exp \left(-\lambda_{n} / y\right),
$$

which is easily shown by induction on $m$, we deduce that

$$
\begin{aligned}
y^{-m-1} \sum a(n) \lambda_{n}^{m} \exp \left(-\lambda_{n} / y\right)= & m ! y^{r-1} \sum b(n) \exp \left(-\mu_{n} y\right) L_{m}^{(r-1)}\left(\mu_{n} y\right) \\
& +\frac{1}{2 \pi i} \int_{C} \frac{\chi(s) \Gamma(s+m) y^{s-1}}{\Gamma(s)} d s .
\end{aligned}
$$

Upon the replacement of $y$ by $1 / y,(10.1)$ follows.

Proof of Theorem 10.2. Using (1.4), we have

$$
\begin{aligned}
\int_{0}^{\infty} e^{-y x} A_{q}(x) d x & =\frac{1}{\Gamma(q+1)} \sum a(n) \exp \left(-\lambda_{n} y\right) \int_{0}^{\infty} e^{-y x} x^{q} d x \\
& =y^{-q-1} \Phi(y) \\
& =y^{-r-q-1} \Psi(1 / y)+y^{-q-1} P(y) .
\end{aligned}
$$

From (2.22) one can show that

$$
d^{m}\left(y^{-r-q-1} \exp \left(-\mu_{n} / y\right)\right) / d y^{m}=(-1)^{m} m ! y^{-m-r-q-1} \exp \left(-\mu_{n} / y\right) L_{m}^{(r+q)}\left(\mu_{n} / y\right) .
$$

Thus, differentiation of both sides of (10.4) $m$ times with respect to $y$ yields

$$
\begin{aligned}
\int_{0}^{\infty} e^{-y x} x^{m} A_{q}(x) d x= & m ! y^{-m-r-q-1} \sum b(n) \exp \left(-\mu_{n} / y\right) L_{m}^{(r+q)}\left(\mu_{n} / y\right) \\
& +\frac{1}{2 \pi i} \int_{C} \frac{\chi(s) \Gamma(s+m+q+1) y^{-s-m-q-1}}{\Gamma(s+q+1)} d s .
\end{aligned}
$$

However, a direct calculation shows that

$$
\int_{0}^{\infty} e^{-y x} \cdot x^{m} Q_{q}(x) d x=\frac{1}{2 \pi i} \int_{C} \frac{\chi(s) \Gamma(s+m+q+1) y^{-s-m-q-1}}{\Gamma(s+q+1)} d s,
$$

provided that $\operatorname{Re} s+q+m+1>0$. Combining (10.5) and (10.6), we establish (10.2). 


\section{Examples.}

EXAMPLE 11.1. Let $\chi$ be a nonprincipal, primitive character modulo $k$ with $\chi(-1)=-1$. Then, the Dirichlet $L$-function $L(s, \chi)$ satisfies the functional equation

$$
\xi(s, \chi)=\varepsilon(\chi) \xi(1-s, \bar{\chi})
$$

where

$$
\xi(s, \chi)=(\pi / k)^{-(s+1) / 2} \Gamma(\{s+1\} / 2) L(s, \chi)
$$

and $|\varepsilon(\chi)|=1$. Thus, $\varphi(s)=(\pi / k)^{-(s+1) / 2} L(s, \chi)$ satisfies Theorem 3.2 with $\lambda_{n}=\mu_{n}$ $=(\pi / k)^{1 / 2} n, \quad a(n)=(\pi / k)^{-1 / 2} \chi(n)$ and $b(n)=\varepsilon(\chi)(\pi / k)^{-1 / 2} \bar{\chi}(n)$. Choose $\gamma=1+\varepsilon$, $0<\varepsilon<1 / 2$. In the strip $-\varepsilon<\sigma<1+\varepsilon, \Gamma(s) L(s, \chi)$ has a simple pole at $s=0$. Replacing $x$ by $(\pi / k)^{1 / 2} x$, we find from (i) that for $q \geqq 1$,

$$
\frac{1}{\Gamma(q+1)} \sum_{n \leqq x} \chi(n)(x-n)^{q}=\frac{L(0, \chi) x^{q}}{\Gamma(q+1)}-2 k^{-1 / 2} \varepsilon(\chi) x^{q+1} \sum \bar{\chi}(n) T_{q+1}(2 \pi n x / k) .
$$

In particular, if $q=1$,

$$
\sum_{n \leqq x} \chi(n)(x-n)=L(0, \chi) x-2 k^{-1 / 2} \varepsilon(\chi) \sum \bar{\chi}(n) \frac{\sin (2 \pi n x / k)}{(2 \pi n / k)^{2}}
$$

From (ii) we have for $q \geqq 1$,

$$
\begin{aligned}
& \frac{1}{\Gamma(q+1)} \sum_{n \leqq x} \chi(n) \log ^{q}(x / n) \\
&=\frac{1}{\Gamma(q+1)} \sum_{j=0}^{q}\left(\begin{array}{l}
q \\
j
\end{array}\right) L^{(j)}(0, x) \log ^{q-j} x \\
&+k^{1 / 2} \varepsilon(\chi) \sum \frac{\bar{\chi}(n)}{n} \int_{\pi n \times / k}^{\infty} \frac{\cos (2 u)}{u} s(u, \pi n x / k ; q-1) d u .
\end{aligned}
$$

In particular, if $q=1$,

$$
\begin{aligned}
\sum_{n \leq x} \chi(n) \log (x / n)= & L(0, \chi) \log x+L^{\prime}(0, \chi) \\
& +k^{1 / 2} \varepsilon(\chi) \sum \frac{\bar{\chi}(n)}{n} \int_{\pi n x / k}^{\infty} \frac{\cos (2 u)}{u} d u \\
= & L(0, \chi) \log x+L^{\prime}(0, \chi)+O(1 / x),
\end{aligned}
$$

by Lemma 2.3. By repeated integrations by parts, the $O$-term above may be replaced by an asymptotic expansion.

EXAMPLE 11.2. Let $\chi$ be as in Example 11.1. Then, $\varphi(s)=(\pi / k)^{-(s+1)} L^{2}(s, \chi)$ satisfies Theorem 5.1 with $\lambda_{n}=\mu_{n}=\pi n / k, a(n)=(k / \pi) \sum_{j k=n} \chi(j) \chi(k)$ and

$$
b(n)=(k / \pi) \varepsilon^{2}(\chi) \sum_{j k=n} \bar{\chi}(j) \bar{\chi}(k) .
$$


Choosing $\gamma=1+\varepsilon, 0<\varepsilon<1 / 2$, and replacing $x$ by $\pi x / k$, we may write down identities analogous to those in Example 11.1 for $q \geqq 1$. In particular, (5.4) yields

$$
\begin{aligned}
\sum_{m n \unlhd x} \chi(m) \chi(n) \log (x / m n)= & L^{2}(0, \chi) \log x+2 L(0, \chi) L^{\prime}(0, \chi) \\
& -(k / 2 \pi) \varepsilon^{2}(\chi) \sum \frac{1}{n}\left\{\sum_{j=n} \bar{\chi}(j) \bar{\chi}(k)\right\} \\
& \cdot\left\{Y_{0}\left(4 \pi(n x)^{1 / 2} / k\right)-\frac{2}{\pi} K_{0}\left(4 \pi(n x)^{1 / 2} / k\right)\right\} .
\end{aligned}
$$

EXAMPLE 11.3. Let $\varphi(s)=\psi(s)=\pi^{-s} f(s)=\pi^{-s} \zeta(s) \zeta(s-k)$, where $\zeta(s)$ denotes the Riemann zeta function and $k \neq 0,-1$ is an integer. Then $\varphi$ satisfies the functional equation of Theorem 6.1 with $p=k, \lambda_{n}=\mu_{n}=\pi n$ and $a(n)=b(n)=\sigma_{k}(n)=\sum_{d \mid n} d^{k}$. Replacing $x$ by $\pi x$, we obtain for $q \geqq \sup (1, k+1 / 2)$

$$
\begin{aligned}
\frac{1}{\Gamma(q+1)} \sum \sigma_{k}(n) & \log ^{q}(x / n) \\
= & \frac{\zeta(k+1) x^{k+1}}{(k+1)^{q+1}}+\zeta(1-k) x+\frac{1}{\Gamma(q+1)} \sum_{j=0}^{q}\left(\begin{array}{l}
q \\
j
\end{array}\right) f^{(j)}(0) \log ^{q-j} x \\
& +\frac{2^{q-k-1}}{\pi^{k+1}} \sum \frac{\sigma_{k}(n)}{n^{k+1}} \frac{d^{q-1}}{d x^{q-1}} \\
& \cdot\left(x^{q-1} \int_{2 \pi(n x)^{1 / 2}}^{\infty} u^{k-q+1} F_{q, k}(2 u) s\left(u, 2 \pi(n x)^{1 / 2} ; q-1\right) d u\right) .
\end{aligned}
$$

This extends and corrects a result in [2]. If $k=-1$, we obtain a similar identity. The case $k=0$ corresponds to $d(n)$, and the identity is given in [2].

EXAMPLE 11.4. In Corollary 9.2 put $r=1$. Using (2.21) we find that

$$
\begin{aligned}
\pi^{1 / 2} \sum a(n)\left(\lambda_{n}+a\right)^{-1 / 2} & \exp \left(-2\left\{\left(\lambda_{n}+a\right) b\right\}^{1 / 2}\right) \\
= & \int_{0}^{\infty} x^{-1 / 2} \exp (-a x-b / x) P(x) d x \\
& +\pi^{1 / 2} \sum b(n)\left(\mu_{n}+b\right)^{-1 / 2} \exp \left(-2\left\{\left(\mu_{n}+b\right) a\right\}^{1 / 2}\right) .
\end{aligned}
$$

Let $\phi(s)=(2 \pi /|\Delta|)^{-s} \zeta(s, Q)$, where $\zeta(s, Q)$ denotes Epstein's zeta function associated with the positive definite quadratic form $Q$ in two variables with discriminant $\Delta$ and integral coefficients. Then, $P(x)=\varphi(0)+1 / x=-1+1 / x$. Using (2.10) and (2.21), we find that

$$
\int_{0}^{\infty} x^{-1 / 2} e^{-a x-b / x}(-1+1 / x) d x=\pi^{1 / 2} \exp \left(-2(a b)^{1 / 2}\right)\left(b^{-1 / 2}-a^{-1 / 2}\right) .
$$

Define $a(0)=b(0)=1$ and $\lambda_{0}=\mu_{0}=0$ and replace $a$ by $2 \pi a /|\Delta|$ and $b$ by $2 \pi b /|\Delta|$. 
Since $\lambda_{n}=\mu_{n}=2 \pi n /|\Delta|$ and $a(n)=b(n)=r(n)$, the number of representations of $n$ by $Q,(11.1)$ becomes

$$
\begin{aligned}
\sum_{n=0}^{\infty} r(n)(n+a)^{-1 / 2} \exp ( & \left.-(4 \pi /|\Delta|)\{(n+a) b\}^{1 / 2}\right) \\
= & \sum_{n=0}^{\infty} r(n)(n+b)^{-1 / 2} \exp \left(-(4 \pi /|\Delta|)\{(n+b) a\}^{1 / 2}\right) .
\end{aligned}
$$

This identity is due to Ramanujan and is stated by Hardy without proof at the end of [12], although a proof is indicated. The identity for the special case $Q(m, n)=m^{2}+n^{2}$ is derived in a more complicated way in [9].

\section{REFERENCES}

1. Bruce C. Berndt, Identities involving the coefficients of a class of Dirichlet series. I, Trans. Amer. Math. Soc. 137 (1969), 345-359.

2. Identities involving the coefficients of a class of Dirichlet series. II, Trans. Amer. Math. Soc. 137 (1969), 361-374.

3. - Generalised Dirichlet series and Hecke's functional equation, Proc. Edinburgh Math. Soc. 15 (1967), 309-313.

4. S. Bochner, Some properties of modular relations, Ann. of Math. 53 (1951), 332-363.

5. K. Chandrasekharan and Raghavan Narasimhan, Hecke's functional equation and arithmetical identities, Ann. of Math. 74 (1961), 1-23.

6. - Hecke's functional equation and the average order of arithmetical functions, Acta Arith. 6 (1961), 487-503.

7. - Functional equations with multiple gamma factors and the average order of arithmetical functions, Ann. of Math. 76 (1962), 93-136.

8. E. T. Copson, Theory of functions of a complex variable, Clarendon Press, Oxford, 1935.

9. A. L. Dixon and W. L. Ferrar, Some summations over the lattice points of a circle (I), Quart. J. Math. Oxford Ser. 5 (1934), 48-63.

10. A. Erdélyi, Editor, Tables of integral transforms, Vol. 1, McGraw-Hill, New York, 1954.

11. I. S. Gradshteyn and I. M. Ryzhik, Table of integrals, series, and products, Academic Press, New York, 1965.

12. G. H. Hardy, On the expression of a number as the sum of two squares, Quart. J. Math. Oxford Ser. 46 (1915), 263-283.

13. E. W. Hobson, The theory of functions of a real variable, Vol. II, 2nd ed., Cambridge Univ. Press, Cambridge, 1926.

14. N. S. Koshliakov, Application of the theory of sumformulae to the investigation of a class of one-valued analytical functions in the theory of numbers, Messenger of Math. 58 (1929), 1-23.

15. J. E. Littlewood, Lectures on the theory of functions, Oxford Univ. Press, Oxford, 1944.

16. G. Szegö, Beiträge zur Theorie der Laguerreschen Polynome. II. Zahlentheoretische Anwendungen, Math. Z. 25 (1926), 388-404.

17. E. C. Titchmarsh, The theory of functions, 2nd ed., Clarendon Press, Oxford, 1939.

18. G. N. Watson, Theory of Bessel functions, 2nd ed., Cambridge Univ. Press, Cambridge, 1944.

UNIVERSITY OF ILLINOIS, URBANA, ILLINOIS 